\title{
Honeybee dance-followers respond similarly to dances regardless of their spatial information
}

content

$$
\text { Matthew J. Hasenjager }{ }^{1} \text {, William Hoppitt }{ }^{1} \text {, Ellouise Leadbeater }{ }^{1}
$$

${ }^{1}$ Department of Biological Sciences, Royal Holloway, University of London, Egham, United Kingdom

Correspondence: M. J. Hasenjager, Department of Biology, Royal Holloway, University of London, Egham, TW20 0EX, United Kingdom. Email address: hasenjag27@gmail.com

\section{Abstract}

Honeybees famously use waggle dances to communicate foraging locations to nestmates in the hive, thereby recruiting them to those sites. The decision to dance is governed by rules that, when operating collectively, are assumed to direct foragers to the most profitable locations with little input from potential recruits, who are presumed to respond similarly to any dance regardless of its information content. Yet variation in receiver responses can qualitatively alter collective outcomes. Here, we use network-based diffusion analysis to compare the collective influence of dance information during recruitment to feeders at different distances. We further assess how any such effects might be achieved at the individual level by dance-followers either persisting with known sites when novel targets are distant and/or seeking more accurate spatial information to guide long-distance searches. Contrary to predictions, we found no evidence that dance-followers' responses depended on target distance. While dance information was 
23 always key to feeder discovery, its importance did not vary with feeder distance, and bees were

24 in fact quicker to abandon previously rewarding sites for distant alternatives. These findings

provide empirical support for the longstanding assumption that self-organized foraging by honeybee colonies relies heavily on signal performance rules with limited input from recipients.

Keywords: collective behaviour; honeybee; network-based diffusion analysis; social information; social insects; waggle dances

\section{Introduction}

Living in groups provides opportunities to pool information across multiple individuals in order to make accurate collective decisions (e.g. navigation in homing pigeons [1]; predator avoidance in fish [2]). In the social insects, such decisions are the product of many (often thousands of) individual-level environmental assessments that are shared with nestmates through evolved communication signals. Simple rules that govern the production or longevity of these signals can generate non-linear feedbacks that produce accurate collective decisions [35]. A classic example involves waggle dance-based recruitment to foraging locations in the western honeybee (Apis mellifera), whereby energetically efficient trips elicit more waggle runs on return to the hive $[6,7]$. Closer sites should hence be over-represented on the dancefloor, and thus attract more recruits, relative to distant alternatives that offer resources of similar quality. This straightforward performance rule could thus enable colonies to collectively optimize energetic efficiency without requiring that dance-followers use the spatial information contained in the dance to make any decision about the potential value of the trip that lies ahead of them [3]. 
In the above scenario, dance-followers are expected to respond similarly to any dance, regardless of its content. Yet research over the past decade has revealed the sophisticated ways

47 in which insects acquire, process, store, retrieve, and use information [8], raising the possibility that signal recipients decide how to respond by weighing the costs and benefits of using that information. For example, ants generally ignore trail pheromones in favour of memories, but will switch to trail-following if information indicates that doing so will lead to a higher quality

51 food source [9]. Likewise, experienced honeybee foragers often discount the spatial

52 information contained in dances in favour of returning to known foraging locations [10-12] and may devalue dance information when it repeatedly proves unreliable [13]. Accounting for such individual variation in receiver responses can lead to qualitatively different outcomes in models 55 of collective behaviour $[3,14,15]$.

Here, we use network-based diffusion analysis (NBDA; $[16,17])$ to evaluate the responses of dance-followers to dances that indicate novel close or distant feeders. NBDA can provide an estimate $(s)$ of the influence of each dance circuit followed on a dance-follower, and we propose that this influence may decrease with distance to the target when dance-followers are unfamiliar with the target resource. This is because locating new sites can require multiple

61 search trips and hence significant time costs that potentially increase with distance $[6,18]$. We

62 created pools of unemployed yet motivated foragers and allowed their recruitment to either close or distant feeders, estimating the strength of social transmission through the resulting dance networks. We further monitored behaviour at the individual level to establish the mechanisms by which such collective effects might be achieved, predicting that (i) bees that follow dances for distant target recruitment sites may persist with known sites for longer, 
67 rather than attempting to locate the new food source, and that (ii) the same bees may invest in gaining more accurate location information by following more waggle runs p re-departure $[19,20]$. Finally, we monitored individual dancer behaviour to confirm our expectation, based on previous work $[6,7]$, that closer resources will be over-represented on the dancefloor.

\section{Methods}

\section{Colony housing}

These experiments were carried out on the campus of Royal Holloway, University of London from July - September 2018. Three queen-right honeybee colonies were housed indoors within three-frame observation hives with unrestricted access via tunnels to the outdoors. Colonies contained $2000-3000$ workers, brood, and reserves of pollen and honey. Each colony underwent both a short-distance and a long-distance recruitment trial, performed consecutively to minimise differences in colony and environmental conditions across trials (Table S1).

\section{Training}

Working with a single colony at a time, two groups of foragers (13 - 31 per group) were simultaneously trained using standard techniques (described in $[21,22]$ ) to two feeders providing unscented sucrose solution. In each case, one feeder was designated the recruit feeder (always $100 \mathrm{~m}$ from the hive) and the other the target feeder (either $100 \mathrm{~m}$ or $500 \mathrm{~m}$ from the hive) with an angular separation of $\sim 110^{\circ}$ between the two feeders (figure 1 ). During training, foragers were assigned unique enamel paint marks upon first arriving at a feeder, 

depleted, creating a pool of marked potential recruits for the target feeder (figure 1).

a

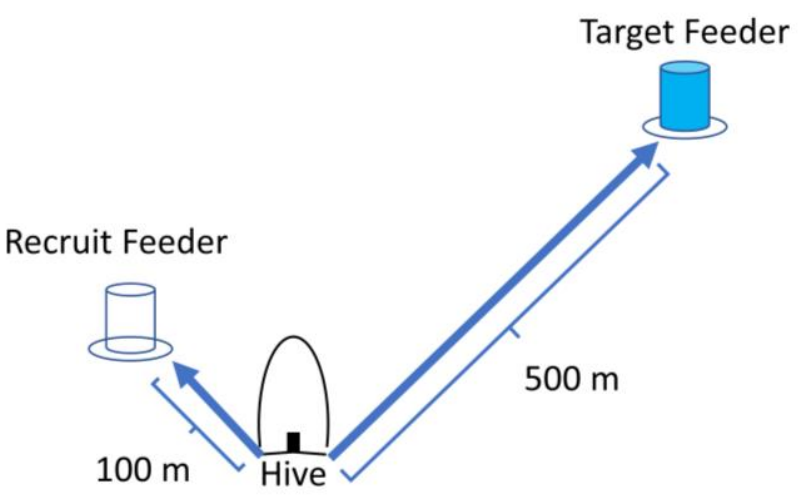

b

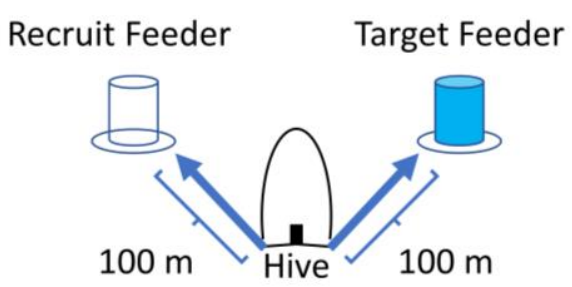

meaning we could be confident that individuals trained to the recruit feeder had never visited the target feeder. Later, during the test period (see below), the recruit feeder would become individuals that visited the recruit feeder during either this odour presentation or during the

Figure 1. Feeder arrangement used during (a) long-distance and (b) short-distance trials. Within each trial, cohorts were simultaneously trained to the recruit and target feeders. During trials, the recruit feeder was left empty to create a pool of potential recruits for the target feeder. Training took place over 5 - 11 days per trial. Both feeders offered identically scented sucrose on the final day of training for one hour (following [22]: $50 \mu \mathrm{L}$ essential oil per $\mathrm{L}$ sucrose, plus reservoir of essential oil below the feeder; scents varied between trials; table S1) in order to promote greater interest in the target feeder during the trial [see 11]. Only previous training day were used as potential recruits during the trials. Although most potential 
103

104

105

106

107

108

109

110

111

112

113

114

115

116

117

118

119

120

121

122

123

124

recruits visited the recruit feeder multiple times during both of these training days, a small

number of individuals only visited the feeder once during the odour presentation or were only

observed during one 30 min census on the previous day. However, as excluding these 21

individuals did not qualitatively alter our findings or conclusions, we retained them in the full

analysis.

\section{Trials}

Trials commenced between 0930 - 1000 on the following morning. During a trial, the target

feeder continued to provide scented $2 \mathrm{M}$ sucrose, whereas the recruit feeder was left empty

(figure 1), thus mimicking a common natural scenario in which one tree or patch of flowers

comes into bloom at the same as another of the same species ceases to be rewarding. We

allowed 10 - 12 foragers previously trained to the target feeder to collect from it, while any

remaining members of this cohort were captured upon arrival. Successful recruits from the

recruit feeder were also allowed to collect freely from the target feeder. We did not restrict the

activities of other bees in the hive, but any that located the target feeder were captured on

arrival. Using both video recordings and in-person observations, we recorded arrival and

departure times for each marked individual at both the recruit and target feeders throughout

the trial. Trials lasted either 120 min (colony A) or 180 min (colonies B and C); this change was

implemented to allow recruits in the $500 \mathrm{~m}$ trials additional time to locate the target feeder.

During trials, we filmed the dance floor within the observation hive. A wooden baffle

directed foragers onto one side of the hive, meaning the vast majority of dances were visible.

For each hive visit made by target feeder foragers (including recruits), we recorded its duration, 
125 whether dancing occurred, and the number of waggle runs produced. We also recorded all dance-following interactions between marked individuals, noting participant identities, when

127 each interaction occurred, its duration (sec), and the number of waggle runs followed. A bee

128 was defined as following a waggle run if its head was oriented towards the dancer within 1

129 antennal length [12]. We further recorded the occurrence of waggle dances by other bees in

130 the hive for natural food sources, whether these dancers carried pollen, the number of waggle

131 runs produced, and any instances in which a marked individual followed one of these dances.

Network-based diffusion analysis (NBDA)

134 All analyses were carried out in R ver. 4.0.3 [23]. In an NBDA, the strength of social transmission

135 per unit of network connection (e.g. per waggle run followed), relative to individual

exploration, is estimated by the social transmission parameter, $s[16,17]$. Here, we set out to

137 compare estimates of $s$ between the close and distant feeders, based on social networks

138 constructed from our video records of dance-following interactions. Specifically, we used order-

139 of-acquisition diffusion analysis, in which networks are used to predict the order in which

140 individuals acquire a behaviour - here, discovery of the target feeder within each trial [17].

141 Network connections were directed from dancers to followers, and we included models where

142 connections were weighted either by the number of waggle runs followed or the total duration

143 (sec) of dance-following in our candidate model sets (described below). To capture the

144 temporal ordering of dance-following interactions, we used dynamic networks that updated

145 when individuals departed the hive for the target feeder [24]. 

our distance treatments, we fit models in which $s$ was either estimated separately for shortand long-distance trials $\left(s_{\text {Short }} \neq s_{\text {Long }}\right.$ ) or in which $s$ was constrained to be equal across these

149 treatments $\left(s_{\text {Short }}=s_{\text {Long }}\right)$. See the Supplementary Material for more details on specification of the NBDA models and for the complete candidate model set. Due to asymmetry in the uncertainty for parameter estimates, profile likelihood techniques were used to obtain $95 \% \mathrm{Cls}$ [25]. The NBDA was carried out using the NBDA package [26].

155 Prior to seeking out a new feeder, honeybee foragers typically return to known sites (often

157 potential differences in this persistence, and in pre-departure information gathering, when the alternative target feeder is either close or distant, we classified trips where individuals were observed at the recruit feeder as "reactivation" trips. If instead that recruit left the hive for more than 90 seconds and successfully discovered the target feeder or was not observed at either site, it was classified as searching for the target feeder ('search trip'). in each global model, is provided in tables S2 and S3 and summarised here. Our primary analyses focussed on the effects of target distance on follower behaviour in terms of: (i) the number of waggle runs followed before departing the hive (zero-inflated negative binomial absences (binomial GLMM). To confirm that longer target distances incur greater search costs, 
we also analysed (iii) the duration of hive absences (linear mixed-effects model); and (iv) the number of unsuccessful searches prior to locating the target feeder (Poisson GLMM).

For completeness, we also analysed dancer behaviour across the short- and longdistance treatments, to compare how the two target feeders were represented on the dancefloor. We included (i) hive visit frequency (linear mixed-effects model); (ii) mean hive visit duration (linear mixed-effects model); (iii) the probability of dancing per visit (binomial GLMM); and (iv) the mean number of waggle runs produced during visits with dancing (linear mixedeffects model).

In every model, Trial and colony were included as a random intercept term and fixed effect respectively; individual was included as a random effect for analyses that included multiple observationsper individual. All input variables were mean-centred and continuous variables were scaled by dividing by twice their standard deviation $[28,29]$. LMMs were fitted using nlme [30] to model heteroscedasticity in the residuals [31] and GLMMs were fitted with glmmTMB [32]. Inspection of GLMM residuals was carried out using DHARMa [33].

We performed model selection on all candidate models nested within each global model (tables S2 and S3) on the basis of AICc. Models were removed from the candidate set if they were more complex versions of a model with a lower AICc value [29,34,35]. From this reduced model set, we extracted a $95 \%$ confidence set of models and used these to obtain modelaveraged parameter estimates (MAEs), unconditional standard errors (USEs), and unconditional 95\% confidence intervals ( $\mathrm{Cls})$ [34]. Where a single model received especially strong support $\left(w_{i} \geq 0.95\right)$, inferences were based on this model alone. Multimodel inference was performed using the MuMIn package [36]. 


\section{Results}

In the short- and long-distance trials respectively, 49 and 25 recruits successfully located the target feeder (table 1). Dance information was key in guiding to foragers to the target feeder, regardless of its distance from the hive. Of our candidate set for the NBDA, two models received nearly all support (model probabilities: $w_{1}=0.91 ; w_{2}=0.09$ ). Both included the dance-following network and constrained social transmission rates to be equal across distance treatments (i.e. $s_{100 \mathrm{~m}}=S_{500 \mathrm{~m}}$ ), indicating that the acceleratory effects of dance-based transmission over how rapidly individuals discovered the target feeder did not vary with foraging distance. The two models differed only in how network connections were weighted: the top-ranked model weighted connections according to the number of waggle runs followed, whereas the second-ranked model used the total duration of dance-following interactions. The best-supported model estimated a social transmission rate of $2.42 \times 10^{7}$ (95\% Cl: $\left.0.90,+\infty\right)$, corresponding to an estimated $97-100 \%$ of recruitment events explained by dance-following.

NBDA indicated that successful recruitment was predicted by an individual's investment in dance-following but provided no evidence that the influence of dance information differed according to the indicated distance. See table S4 for parameter estimates from both models. 
213 Table 1. Summary of experimental trials (TF: target feeder; RF: recruit feeder). Data provided as 214 sample size or mean \pm SD. Hive absences were labelled as 'reactivation' if a forager returned to 215 the RF; otherwise, foragers were assumed to be searching for the TF.

\begin{tabular}{lllllll}
\hline Colony & $\begin{array}{l}\text { TF } \\
\text { distance }\end{array}$ & $\begin{array}{l}\text { RF } \\
\text { trained }\end{array}$ & $\begin{array}{l}\text { TF } \\
\text { recruits }\end{array}$ & $\begin{array}{l}\text { Dancers: Waggle } \\
\text { runs per hive visit }\end{array}$ & \multicolumn{2}{l}{$\begin{array}{l}\text { Followers: TF waggle runs } \\
\text { followed per hive visit }\end{array}$} \\
\hline & & & & & & \multicolumn{2}{l}{ Reactivation } & Searching \\
\cline { 5 - 6 } & $100 \mathrm{~m}$ & 21 & 16 & $11.4 \pm 18.5$ & $3.9 \pm 4.2$ & $7.4 \pm 5.3$ \\
$\mathrm{~A}$ & $500 \mathrm{~m}$ & 26 & 3 & $11.8 \pm 15.3$ & $2.3 \pm 3.5$ & $8.3 \pm 6.4$ \\
$\mathrm{~B}$ & $100 \mathrm{~m}$ & 31 & 22 & $15.5 \pm 15.5$ & $5.2 \pm 6.9$ & $14.4 \pm 7.9$ \\
B & $500 \mathrm{~m}$ & 22 & 9 & $12.5 \pm 13.6$ & $3.6 \pm 4.6$ & $17.4 \pm 10.2$ \\
$\mathrm{C}$ & $100 \mathrm{~m}$ & 28 & 11 & $4.6 \pm 8.1$ & $2.3 \pm 3.3$ & $4.7 \pm 4.8$ \\
C & $500 \mathrm{~m}$ & 30 & 13 & $11.3 \pm 13.6$ & $4.1 \pm 7.1$ & $13.6 \pm 8.5$ \\
\hline
\end{tabular}

\section{Follower behaviour}

218 As expected, individuals typically made multiple trips to the empty recruit feeder before

219 searching for the new target (see also [11]), and the probability of abandoning the recruit

220 feeder in favour of searching for the target feeder increased over time (binomial GLMM: hive

221 visit: MAE \pm USE $=2.7 \pm 0.23(95 \% \mathrm{Cl}: 2.25,3.15)$; figure 2). However, contrary to our

222 expectations, bees were quicker to engage in search trips when the target feeder was distantly

223 located than when it was close to the hive (binomial GLMM: target feeder distance (500 m):

224 MAE \pm USE $=0.9 \pm 0.22(95 \% \mathrm{Cl}: 0.48,1.32)$; target feeder distance ${ }^{*}$ hive visit: $\mathrm{MAE} \pm$ USE $=$

$2251.91 \pm 0.46(95 \% \mathrm{Cl}: 1.01,2.81)$; figure 2). See tables S5 and S6 for full model summaries. 


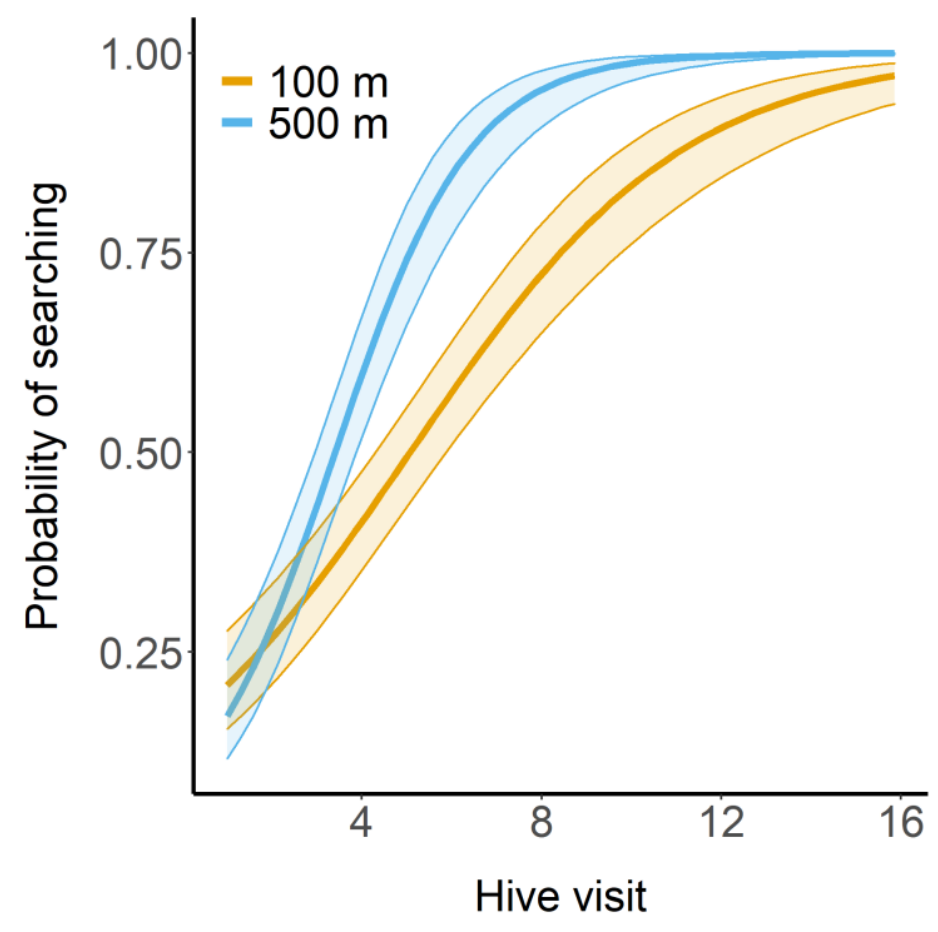

227 Figure 2. Predicted probability of searching for the target feeder upon departing the hive. Lines and shaded regions respectively indicate predicted values and $95 \% \mathrm{Cl}$ calculated from modelaveraged GLMM fixed effects with all random effects set to 0 . waggle runs before departing in search of the target feeder than when re-visiting the empty feeder, though this effect lessened over time (zero-inflated negative binomial GLMM: search trip: MAE \pm USE $=0.75 \pm 0.06(95 \% \mathrm{Cl}: 0.63,0.87)$; searching * hive visit: MAE \pm USE $=-0.53 \pm$ $0.13(95 \% \mathrm{Cl}:-0.78,-0.27))$. However, there was no evidence at the $95 \%$ confidence level that bees followed more waggle runs before searching when the feeder was more distantly located (same GLMM: TF distance $(500 \mathrm{~m})$ * search trip: MAE \pm USE $=0.17 \pm 0.15(95 \% \mathrm{Cl}:-0.11,0.47)$;

237 figure 3). See tables S7 and S8 for full model summaries. 


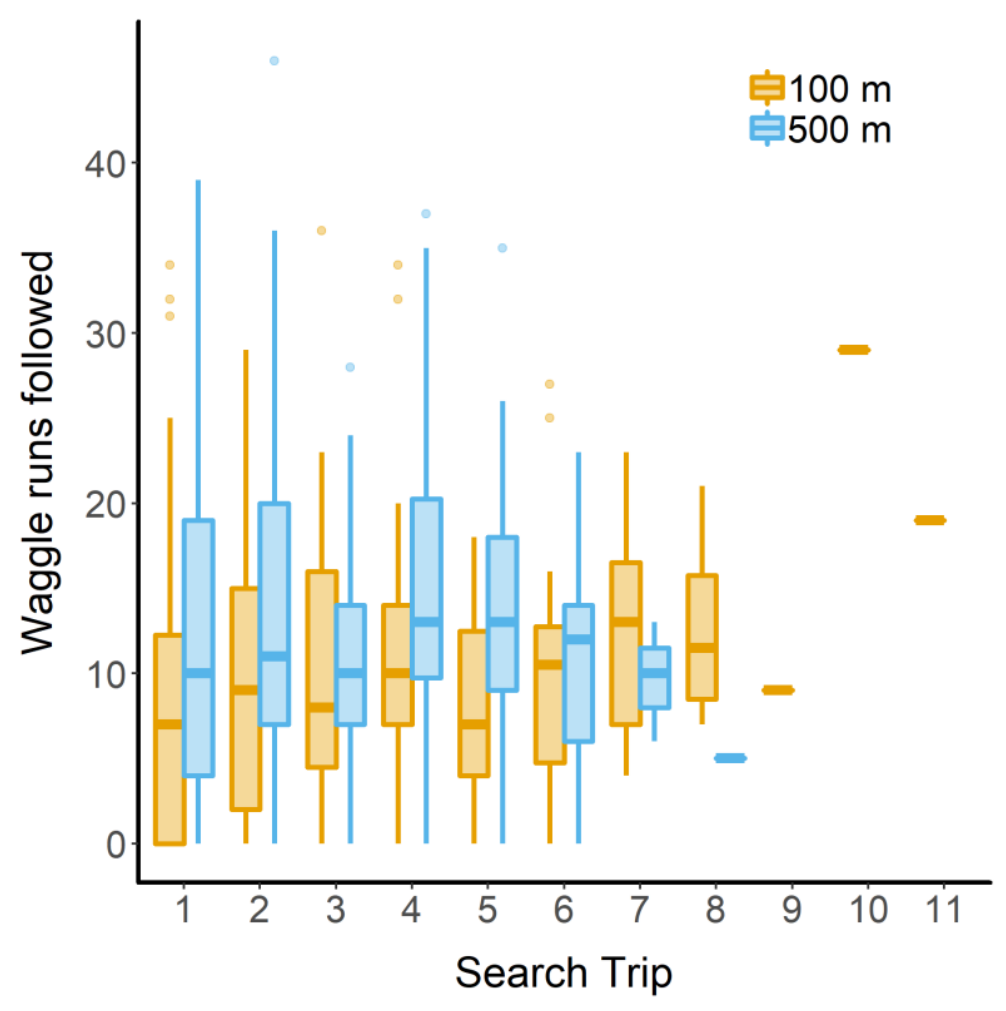

Figure 3. The number of waggle runs followed prior to searching for the target feeder. Thick lines indicate medians, boxes enclose the interquartile range, and whiskers extend to up to $1.5 x$ this range.

As expected if long-distance searches are more costly, search trips into the field were longer in duration than reactivation trips only when the target feeder was more distant (LMM: target feeder distance $(500 \mathrm{~m})$ * search trip: MAE \pm USE $=0.6 \pm 0.06(95 \% \mathrm{Cl}: 0.48,0.72)$; figure 4a; tables S9 \& S10). Comparing the mean duration of searches for the target feeder vs. collection trips made by employed foragers (minus time spent at the feeder) confirmed that both search and collection trips took more time when the target feeder was more distant from the hive (LMM: target distance $(500 \mathrm{~m})$ : estimate $\pm \mathrm{SE}=0.63 \pm 0.03(95 \% \mathrm{Cl}: 0.5,0.76)$; table S11) and that search trips were longer in duration than collection trips (LMM: trip type (collection): estimate \pm SE $=-0.6 \pm 0.05(95 \% \mathrm{Cl}:-0.69,-0.51)$; table S11)). However, searches 
251 were not disproportionately longer at $500 \mathrm{~m}$ than at $100 \mathrm{~m}$ (the best-supported model, $w_{i}>$

252 0.99, did not include an interaction between target feeder distance and trip type; table S11).

253 Regardless of distance, successful recruits undertook a similar number of unsuccessful searches

254 before eventually locating the target feeder (Poisson GLMM: target feeder distance (500 m):

255 MAE \pm USE $=-0.24 \pm 0.22$ (95\% Cl: $-0.67,0.18)$; figure $4 b$; tables S12 \& S13).

256 In addition to dances for the target feeder, we observed 122 dances for natural food

257 sources. These dancers produced $8.74 \pm 12.37$ (mean \pm SD) waggle runs per dance and carried

258 pollen in 56 of these dances. Although our focal bees occasionally followed these natural

259 dances, these following events were brief in duration (mean \pm SD $=1.13 \pm 0.35$ waggle runs

260 followed; $n=40$ dance-following events). Out of 519 prospective search flights, only 10 (i.e.

261 1.9\%) involved a focal bee following a dance for a natural food source (mean \pm SD $=1.8 \pm 1.03$

262 waggle runs followed). 
a

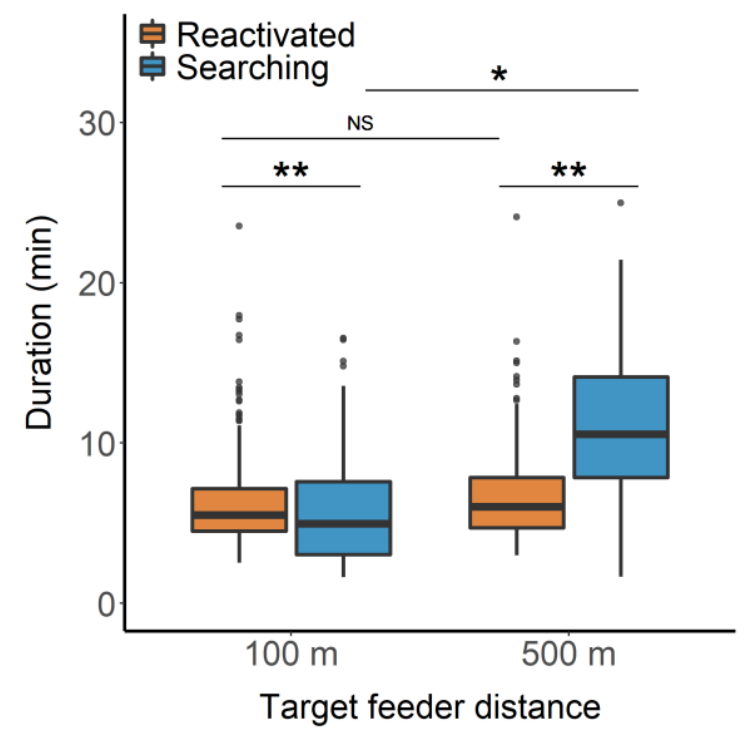

B

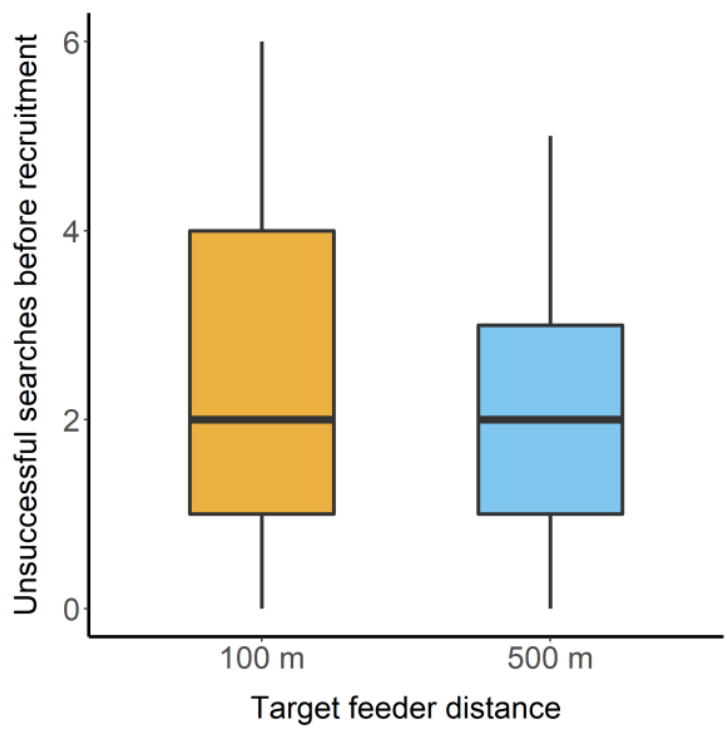

Figure 4. (a) Duration of hive absences and (b) number of unsuccessful searches before

264 discovering the target feeder. Absences were labelled as reactivations if foragers returned to

265 the empty recruit feeder and searches for the target feeder otherwise. The y-axis in (a) has

266 been truncated to enhance clarity; an additional reactivation was observed in both the short-

267 and long-distance trials with respective durations of 64.9 and $35.7 \mathrm{~min}$. Thick lines indicate

268 medians, boxes enclose the interquartile range, and whiskers extend up to $1.5 x$ this range. $P$

269 values for contrasts were adjusted using the Bonferroni method: *: $\mathrm{P}<0.01 ;{ }^{* *}: \mathrm{P}<0.001$. 
a

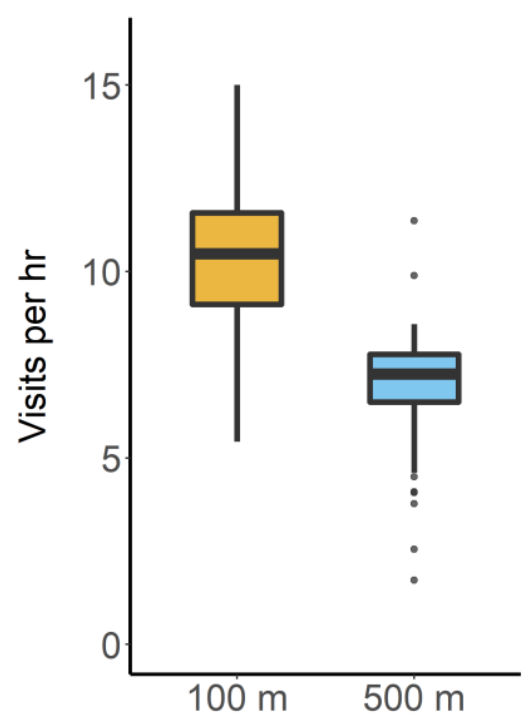

Feeder distance b

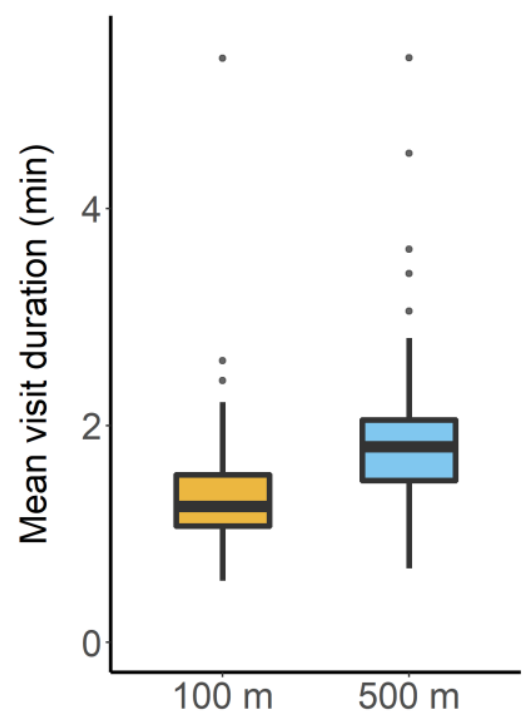

Feeder distance
C

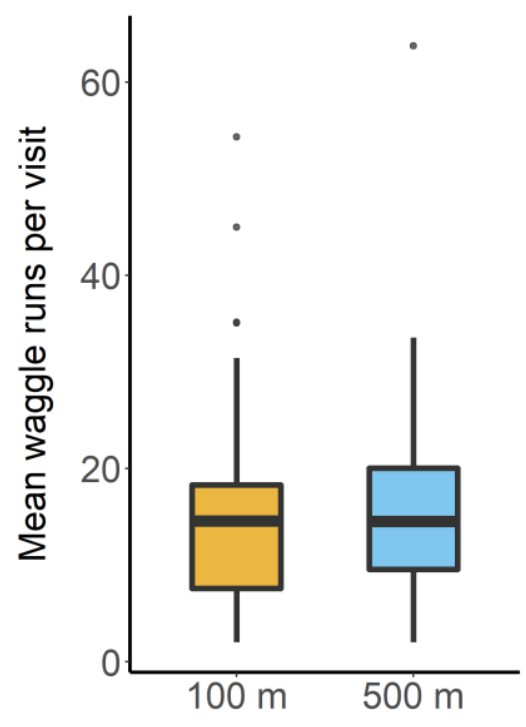

Feeder distance

271 Figure 5. (a) Hive visit frequency, (b) mean visit duration, and (c) mean waggle runs produced

272 per visit with dancing by foragers collecting from the target feeder. Thick lines indicate

273 medians, boxes enclose the interquartile range, and whiskers extend up to $1.5 x$ this range.

\section{Dancer behaviour}

In line with previous work [7,21], dances representing the more distant (and thus less

277 energetically efficient) $500 \mathrm{~m}$ feeder were underrepresented on the dancefloor relative to

278 those for the closer feeder. This occurred because dancers visited the hive less frequently when

279 the target feeder was more distantly located (LMM: target feeder distance (500 m): estimate \pm

$280 \mathrm{SE}=-3.58 \pm 0.37(95 \% \mathrm{Cl}:-5.15,-2.01) ;$ table S14). On average, dancers made 10.3 visits $\mathrm{hr}^{-1}$

281 when the feeder was located $100 \mathrm{~m}$ from the hive, but only 6.9 visits $\mathrm{hr}^{-1}$ when it was $500 \mathrm{~m}$ 
S11), but foragers collecting at $500 \mathrm{~m}$ also tended to remain in the hive for longer on each visit (LMM: target feeder distance $(500 \mathrm{~m})$ : estimate $\pm \mathrm{SE}=0.51 \pm 0.09$ (95\% Cl: 0.12, 0.89); figure $5 b$; table S15). In contrast to our expectation that bees foraging at the distant feeder would be less likely to dance upon returning to the hive, there was no evidence that foraging distance influenced foragers' propensity to dance (table S16). If anything, foragers in long-distance trials tended to be more likely to dance during hive visits (binomial GLMM: target feeder distance $(500 \mathrm{~m}):$ estimate $\pm \mathrm{SE}=0.72 \pm 0.71(95 \% \mathrm{Cl}:-0.67,2.11))$, though the best-supported model $\left(w_{i}\right.$ $>0.99$ ) did not include this effect (table S16). There was also no evidence that dancers for more distant feeders produced fewer waggle runs (LMM: target feeder distance (500 m): MAE \pm USE $=0.31 \pm 1.79$ (95\% Cl: $-3.2,3.82) ;$ tables S17 \& S18; figure 5c).

\section{Discussion}

The traditional view of insects as mere stimulus-response "machines" has given way to a growing recognition that despite their miniature brains, insects possess sophisticated cognitive capabilities [8]. Thus, although empirically derived theoretical models have shown how simple rules that govern the production of waggle dances are sufficient to generate adaptive collective responses by honeybee colonies without requiring that dance-followers evaluate the transmitted spatial information $[3,22]$, dance-followers may in principle be able to fine-tune their responses according to this information. Here, we used NBDA to first ask whether bees respond differently to dances depending on the indicated foraging distance. In contrast to our predictions, we found no difference in the estimated influence of dance communication ( $s$ ) on the order in which recruits arrived at our close or distant novel feeders. We further found that 
increased foraging distance was not associated with increased investment in dance-following prior to searching, and that foragers were in fact quicker to abandon a depleted site when the alternative was more distantly located. Taken together, these findings suggest that dancefollowers do not evaluate the distance information contained in a dance when deciding how to respond to it.

As foraging distances increase, searches require progressively greater investments in time and energy, exacerbated by the fact that dance-guided searches often fail (figure 4b; $[6,18])$. Why then are bees not more reticent to accept recruitment to distant novel sources? Dances are followed both by bees that have never visited the target site (recruitment) and bees that know its location (reactivation), with only the former incurring search costs. Since dancers do not know for which purpose their audience is following, we expected the behavioural rules that translate the energetic efficiency of a foraging trip into the number of waggle runs performed to ignore these additional search costs, allowing instead for dance-followers to finetune their responses depending on their informational status. However, it may be that the increasing search costs elicited by distant resources are already sufficiently accounted for through their under-representation on the dancefloor and that additional receiver responses are not needed to achieve adaptive collective foraging, especially given that we found that although search costs do increase with distance, they are not disproportionately large at greater distances.

Alternatively, it may be the case that while sensitivity to distance information by followers could increase colony foraging efficiency, the mechanisms by which it could be achieved have diminishing returns. For example, beyond a certain point, the extra time spent 
327 following additional dance circuits may not appreciably increase the likelihood of locating a site.

328 Moreover, the positive relationship between foraging site distance and waggle run duration

329 means that as foraging distances increase, foragers must invest ever more time in dance-

330 following to acquire similar amounts of information [21,38]. The use of dance information may

331 involve a speed-accuracy trade-off [39], such that setting out with reasonably accurate spatial

332 information may often be preferable to investing further time in waiting for and following

333 dances.

$10 \mathrm{~km}$ from the hive $[22,40]$, dance decoding studies have shown that the median distance

travelled under natural conditions is often an order of magnitude lower than this [41]. For

example, we recently found the median distance indicated by dances across an entire season in

southern England to be $708 \mathrm{~m}$ and $1108 \mathrm{~m}$ for urban and agricultural sites respectively [42].

Nonetheless, it is clear that our feeder locations, at 100 and 500m from the hive, do not

represent the full foraging range. However, we note that previous work has detected

347 feeder several times before searching for the target feeder. Yet rather than foragers being 
opposite pattern was observed (figure 2). It is possible that this finding simply stems from how foraging trips were labelled-i.e., during reactivations, it was assumed that bees did not also search for the target feeder. However, studies using harmonic radar to track bees' foraging flights have revealed the occurrence of such cross-trips between familiar and unfamiliar foraging locations [43], potentially allowing individuals to gain up-to-date information on familiar foraging sites while also making use of dance information without requiring that they first return to the hive. If joint reactivation-search trips occurred more often in short-distance trials when feeders were relatively close together [43], this could be reflected in our analysis as a lower likelihood of searching when the target was nearby. However, although we cannot rule out that such trips occurred, our data suggest that they were unlikely to be especially common (see Supplementary Material, tables S19 \& S20). Alternately, the dance-indicated location in long-distance trials may have been easier to identify as a novel site, as neither the distance nor directional components matched that of the recruit feeder [21,37]. Regardless, our results complement previous reports that honeybees' persistence to familiar sites depend more on previous profitability than on the availability of alternatives [27].

Although we assumed that during departures from the hive, potential recruits were either returning to the recruit feeder or searching for the target feeder, individuals may also have engaged in alternative foraging behaviours, including visiting other known foraging locations or searching for natural food sources. However, trials took place during the late summer and early autumn when few natural food sources are available to bees in southern England [41]. Accordingly, foragers were highly persistent in visiting the feeders during training, limiting their opportunities to learn about other foraging sites prior to the trial. In addition, 
371 there were relatively few dances for natural sources during the trials and these were rarely

372 followed by our focal individuals. When natural dances were followed, these bouts were always

373 brief in duration, indicating that foragers were not attempting to decode the dance's spatial

374 information [44]. Individuals may also have attempted to locate other foraging sites through

375 individual scouting. However, previous reports have found that scouting is relatively rare when

376 dances are readily available in the hive [18,45], as was the case in our study. We therefore feel

377 confident that most searching events represented attempts to locate the target feeder.

378 Nevertheless, we repeated our analysis of: (i) the duration of searching events and (ii) the

379 number of waggle runs followed prior to each search using only the subset of successful

380 recruitment events. Our findings were consistent with our more inclusive analysis: in long-

381 distance trials, searches were longer in duration and recruits followed more waggle runs prior

382 to a successful search, but this latter difference was not significant at the $95 \%$ level (tables S21

$383 \& \mathrm{~S} 22)$

Given that the colony represents the reproductive unit in honeybees, natural selection is

expected to have acted on the heuristics that guide behaviour at the individual level in order to

produce adaptive colony-level responses [3]. Although such individual-levelalgorithms could in

principal lead recruits to differentially respond to dances according to the indicated distance,

we found no evidence that this is the case. Rather, our results provide empirical support to the 
bees foraging on natural sources, most studies (including our own) have used artificial food sources located relatively near to the hive that offer an unrestricted flow of sucrose. Additional investigations into how the production of dances is modulated under more naturalistic foraging conditions and how dance-followers respond to this information would be worthwhile.

Data accessibility. Raw data and code to reproduce all analyses are available from the Dryad Digital Repository: https://doi.org/10.5061/dryad.8kprr4xn8 [46].

Authors' contributions. M.J.H. and E.L. designed the study. M.J.H. collected the data and M.J.H. and W.H. analysed it. M.J.H. wrote the initial draft and all authors contributed to revisions.

Acknowledgements. We thank Alex Hadleigh for assistance with carrying out the experiments described here.

Competing interests. We declare we have no competing interests.

Funding. This research was funded by the European Research Council under the European Union's Horizon 2020 research and innovation programme (grant number 638873).

\section{References}

1. Dell'Ariccia, G., Dell'Omo, G., Wolfer, D. P., \& Lipp, H.-P. (2008). Flock flying improves pigeons' homing: GPS track analysis of individual flyers versus small groups. Animal Behaviour, 76, 1165-1172.

2. Ward, A. J. W., Herbert-Read, J. E., Sumpter, D. J. T., \& Krause, J. (2011). Fast and accurate decisions through collective vigilance in fish shoals. Proceedings of the National Academy of Sciences of the U. S. A., 108, 2312-2315.

3. Detrain, C., \& Deneubourg, J.-L. (2008). Collective decision-making and foraging patterns in ants and honeybees. Advances in Insect Physiology, 35, 123-173.

4. Seeley, T. D. (2010). Honeybee democracy. Princeton, NJ: Princeton University Press. 
5. Sasaki, T., Granovskiy, B., Mann, R. P., Sumpter, D. J. T., \& Pratt, S. C. (2013). Ant colonies outperform individuals when a sensory discrimination task is difficult but not when it is easy. Proceedings of the National Academy of Sciences of the U. S. A., 110, 13769-13773.

6. Seeley, T. D., \& Towne, W. F. (1992). Tactics of dance choice in honey bees: do foragers compare dances? Behavioral Ecology and Sociobiology, 30, 59-69.

7. Seeley, T. D. (1994). Honey bee foragers as sensory units of their colonies. Behavioral Ecology and Sociobiology, 34, 51-62.

8. Giurfa, M. (2015). Learning and cognition in insects. Wiley Interdisciplinary Reviews: Cognitive Science, 6, 383-395.

9. Czaczkes, T. J., Beckwith, J. J., Horsch, A.-L., \& Hartig, F. (2019). The multi-dimensional nature of information drives prioritization of private over social information in ants. Proceedings of the Royal Society B, 286, 20191136.

10. Grüter, C., Balbuena, M. S., \& Farina, W. M. (2008). Informational conflicts created by the waggle dance. Proceedings of the Royal Society B, 275, 1321-1327.

11. Grüter, C., \& Ratnieks, F. L. W. (2011). Honeybee foragers increase the use of waggle dance information when private information becomes unrewarding. Animal Behaviour, 81, 949954.

12. Hasenjager, M. J., Hoppitt, W., \& Leadbeater, E. (2020). Network-based diffusion analysis reveals context-specific dominance of dance communication in foraging honeybees. Nature Communications, 11, 625 .

13. I'Anson Price, R., Dulex, N., Vial, N., Vincent, C., \& Grüter, C. (2019). Honeybees forage more successfully without the "dance language" in challenging environments. Science Advances, 5, eaat0450.

14. Schürch, R., \& Grüter, C. (2014). Dancing bees improve colony foraging success as long-term benefits outweigh short-term costs. PLOS ONE, 9, e104660.

15. Lemanski, N. J., Cook, C. N., Smith, B. H., \& Pinter-wollman, N. (2019). A multiscale review of behavioral variation in collective foraging behavior in honey bees. Insects, 10, 370.

16. Franz, M., \& Nunn, C. L. (2009). Network-based diffusion analysis: a new method for detecting social learning. Proceedings of the Royal Society $B, 276,1829-1836$. 
17. Hoppitt, W., Boogert, N. J., \& Laland, K. N. (2010). Detecting social transmission in networks. Journal of Theoretical Biology, 263, 544-555.

18. Biesmeijer, J. C., \& Seeley, T. D. (2005). The use of waggle dance information by honey bees throughout their foraging careers. Behavioral Ecology and Sociobiology, 59, 133-142.

19. Tanner, D., \& Visscher, K. (2008). Do honey bees average directions in the waggle dance to determine a flight direction? Behavioral Ecology and Sociobiology, 62, 1891-1898.

20. Tanner, D., \& Visscher, K. (2009). Does the body orientation of waggle dance followers affect the accuracy of recruitment? Apidologie, 40, 55-62.

21. von Frisch, K. (1967). The dance language and orientation of bees. Cambridge, MA: Harvard University Press.

22. Seeley, T. D. (1995). The wisdom of the hive: The social physiology of honey bee colonies. Cambridge, MA: Harvard University Press.

23. $R$ Core Team. (2020). R: a language and environment for statistical computing. $R$ Foundation for Statistical Computing, Vienna, Austria. https://www.R-project.org/

24. Hasenjager, M. J., Leadbeater, E., \& Hoppitt, W. (2021). Detecting and quantifying social transmission using network-based diffusion analysis. Journal of Animal Ecology, 90, 8-26. doi: 10.1111/1365-2656-13307.

25. Morgan, B. J. T. (2009). Applied stochastic modelling ( $2^{\text {nd }}$ ed.) Boca Raton, FL: Chapman \& Hall/CRC Press.

26. Hoppitt, W., Photopoulou, T., Hasenjager, M., \& Leadbeater, E. (2020). NBDA: a package for implementing network-based diffusion analysis. R package version 0.9.4. https://github.com/whoppitt/NBDA

27. Al Toufailia, H., Grüter, C., \& Ratnieks, F. L. W. (2013). Persistence to unrewarding feeding locations by honeybee foragers (Apis mellifera): the effects of experience, resource profitability and season. Ethology, 119, 1096-1106.

28. Schielzeth, H. (2010). Simple means to improve the interpretability of regression coefficients. Methods in Ecology and Evolution, 1, 103-113. 
507

508

509

510

511

512

29. Grueber, C. E., Nakagawa, S., Laws, R. J., \& Jamieson, I. G. (2011). Multimodel inference in ecology and evolution: challenges and solutions. Journal of Evolutionary Biology, 24, 699711.

30. Pinheiro, J., Bates, D., DebRoy, S., Sarkar, D., \& R Core Team. (2018). nlme: linear and nonlinear mixed effects models. R package version 3.1-137, https://CRAN.Rproject.org/package=nIme.

31. Zuur, A. F., leno, E. N., Walker, N. J., Saveliev, A. A., \& Smith, G. M. (2009). Mixed effects models and extensions in ecology with R. New York, NY: Springer Science+Business Media.

32. Brooks, M. E., Kristensen, K., van Benthem, K. J., Magnusson, A., Berg, C. W., Nielsen, A., et al. (2017). glmmTMB balances speed and flexibility among packages for zero-inflated generalized linear mixed modeling. The $R$ Journal, 9, 378-400.

33. Hartig, F. (2020). DHARMa: residual diagnostics for hierarchical (multi-level/mixed) regression models. $\mathrm{R}$ package version 0.3.1. https://CRAN.R-project.org/package=DHARMa.

34. Burnham, K. P., \& Anderson, D. R. (2002). Model selection and multimodel inference: $A$ practical information-theoretic approach ( $2^{\text {nd }}$ Ed.). New York, NY: Springer-Verlag.

35. Richards, S. A. (2008). Dealing with overdispersed count data in applied ecology. Journal of Applied Ecology, 45, 218-227.

36. Barton, K. (2019). MuMIn: multi-model inference. R package version 1.43.6. https://CRAN.Rproject.org/package=MuMIn.

37. Grüter, C., Segers, F. H. I. D., \& Ratnieks, F. L. W. (2013). Social learning strategies in honeybee foragers: do the costs of using private information affect the use of social information? Animal Behaviour, 85, 1443-1449.

38. Al Toufailia, H., Couvillon, M. J., Ratnieks, F. L. W., \& Grüter, C. (2013). Honey bee waggle dance communication: signal meaning and signal noise affect dance follower behaviour. Behavioral Ecology and Sociobiology, 67, 549-556.

39. Chittka, L., Skorupski, P., \& Raine, N. E. (2009). Speed-accuracy tradeoffs in animal decision making. Trends in Ecology and Evolution, 24, 400-407.

40. Beekman, M., \& Ratnieks, F. L. W. (2000). Long-range foraging by the honey-bee, Apis mellifera L. Functional Ecology, 14, 490-496. 
546

547

548

549

550

551

552

553

554

555

556

557

558

559

41. Couvillon, M. J., Schürch, R., \& Ratnieks, F. L. W. (2014). Waggle dance distances as integrative indicators of seasonal foraging challenges. PLOS ONE, 9, e93495.

42. Samuelson, A. E., Schürch, R., \& Leadbeater, E. (2019). Dancing bees evaluate agricultural forage resources as inferior to central urban land. bioRxiv, 2019.12.19.882076.

43. Menzel, R., Kirbach, A., Haass, W.-D., Fischer, B., Fuchs, J., Koblofsky, M., et al. (2011). A common frame of reference for learned and communicated vectors in honeybee navigation. Current Biology, 21, 645-650.

44. Grüter, C., \& Farina, W. M. (2009). The honeybee waggle dance: can we follow the steps? Trends in Ecology and Evolution, 24, 242-247.

45. Beekman, M., Gilchrist, A. L., Duncan, M., \& Sumpter, D. J. T. (2007). What makes a honeybee scout? Behavioral Ecology and Sociobiology, 61, 985-995.

46. Hasenjager, M. J., Hoppitt, W., \& Leadbeater, E. (2021). Data from: Honeybee dancefollowers respond similarly to dances regardless of their spatial information content. Dryad Digital Repository. https://doi.org/10.5061/dryad.8kprr4xn8 